\title{
Lean And Efficient System Software Product Lines: Where Aspects Beat Objects ${ }^{\star}$
}

\author{
Daniel Lohmann, Olaf Spinczyk, Wolfgang Schröder-Preikschat \\ $\{d l$, os, wosch $\}$ ecs.fau.de \\ Friedrich-Alexander-University Erlangen-Nuremberg, Germany \\ Computer Science 4 - Distributed Systems and Operating Systems
}

\begin{abstract}
Software development in the domain of embedded and deeply embedded systems is dominated by cost pressure and extremely limited hardware resources. As a result, modern concepts for separation of concerns and software reuse are widely ignored, as developers worry about the thereby induced memory and performance overhead. Especially object-oriented programming (OOP) is still little in demand. For the development of highly configurable fine-grained system software product lines, however, separation of concerns $(\mathrm{SoC})$ is a crucial property. As the overhead of object-orientation is not acceptable in this domain, we propose aspect-oriented programming (AOP) as an alternative. Compared to OOP, AOP makes it possible to reach similar or even better separation of concerns with significantly smaller memory footprints. In a case study for an embedded system product line the memory costs for SoC could be reduced from 148-236\% to $2-10 \%$ by using AOP instead of OOP.
\end{abstract}

\section{Introduction}

The domain of embedded and deeply embedded devices is dominated by 8 bit $\mu$ controllers with $0.25-4 \mathrm{~KB}$ of RAM and a few KB of flash memory. In 2000, more than $98 \%$ of the total worldwide CPU production ( 8 billion entities) were dedicated to the domain of embedded systems; $87 \%$ of the entities used in this domain were 8 bit or smaller[47]. From the viewpoint of procurement, this "old-fashioned technology" is still the best compromise with respect to functionality and costs. In areas of mass production a few cents decide over market success or failure - a situation that can not be expected to change soon, given that the envisioned scenarios of smart dust[30], ubiquitous computing[48] and proactive computing[47]) crucially depend on the bulk availability of very cheap, self-organizing "intelligent" devices.

Counting cents in hardware procurement basically leads to counting bytes in software development. To cope with hardware cost pressure and extremely limited resources, software developers for deeply embedded systems intentionally avoid modern language concepts for a better separation of concerns (SoC), as they worry about the thereby induced memory and performance overhead. Especially object-oriented programming (OOP) is still little in demand, as some of its fundamental concepts (e.g. late

\footnotetext{
* This work was partly supported by the German Research Council (DFG) under grant no.
} SCHR 603/4 and SP 968/2-1. 
binding by virtual functions) are known to have non-negligible costs[20]. Hence, most software development for embedded systems is still performed "ad-hoc" in C (often even assembler) with a strong focus on minimizing hardware requirements. Organized reuse and separation of concerns is often considered as less important.

\section{System Software Product Lines}

The common "ad-hoc" application-specific development approach is doomed to fail for reusable system software, such as operating systems or light-weight middleware for embedded devices. System software for this domain has not only to cope with the above mentioned resource constraints, but also with a very broad variety of functional and non-functional requirements[10]. It has to be tailorable to provide exactly the functionality required by the intended application, but nothing more. This leads to a familybased or product line approach, where the variability and commonality among family members is expressed by feature models[16]. The tailorability of software product lines depends mostly on the offered level of functional exchangeability (here denoted as variability) and functional selectability (here denoted as granularity). Both, variability and granularity, require a (right-unique) mapping from implementation components to the features they implement, thus, a good separation of concerns. As the principal overhead of OOP is not acceptable in this domain, we advocate to use aspect-oriented programming $(A O P)$ instead.

AOP, as well as OOP, provides means for a better separation of concerns. Today, AOP is mostly perceived as an extension to OOP, which leads to the impression that it has to induce similar or even higher overhead. This is understandable, as most aspect languages are actually extensions to object-oriented languages like Java. For AspectJ[31], studies show furthermore that AOP concepts may indeed lead to some extra overhead [21]. Nevertheless, AOP itself is not limited to OOP. Language extensions have as well been proposed for non-OOP languages (such as AspectC[12]) and multiparadigm languages (such as AspectC++[46]). In these languages it is possible to use AOP not only as extension, but also as alternative to OOP.

\section{Objectives}

This article shows that for the specific requirements of resource-constrained software development, AOP can be superior to OOP. Our focus is not on separation of crosscutting concerns and other structural benefits of AOP that already have been discussed in many papers. Instead, we want to draw attention to a mostly unexplored benefit of AOP: Given a well-optimizing static weaver, AOP can provide SoC more resource-efficiently than OOP. Our goals are in particular to:

- broaden the perception of AOP. Currently AOP is mostly perceived as an extension to OOP for a better SoC. Understanding it as an alternative to OOP with respect to resource-efficiency and hardware costs is a new point of view.

- attract new communities to AOP. Especially the systems and embedded communities have a profound skepticism regarding the suitability of high-level programming paradigms ("everything beyond C"), as they (have to) favor efficiency over SoC. 
Demonstrating that with aspects it can be possible to reach SoC without having to give up efficency would help to broaden the acceptance of AOP.

For this purposes, we conducted a study in which we compare the resource requirements of three implementations of the same embedded software product line. A highly efficient C-based implementation that does not provide SoC, an object-oriented, and an aspect-oriented implementation. We evaluate the $\mathrm{OO}$ and $\mathrm{AO}$ implementations regarding the techniques used to reach SoC. The focus is on the thereby induced costs. While both implementations reach the SoC goals, the OO-based solution leads to significantly higher resource requirements than the AO-based solution, which can compete with plain $\mathrm{C}$.

\section{Structure of the Paper}

The paper is organized as follows: In the next section, we present our case study about an aspect-oriented and an object-oriented implementation of an embedded software product line. Both implementations are analyzed with respect to used idioms and potential cost drivers. In section 4, these implementations are further investigated regarding their resource requirements. The results are discussed in section 5. Section 6 provides an overview of related work. Finally, the paper is briefly summarized in section 7 .

\section{Scenario}

In the following, we present and analyze a case study of an embedded software product line with three different implementations: C-based, OOP-based, and AOP-based. For the sake of comprehensibility, we have conducted this case study in a more applicationoriented field. The scenario is an embedded weather station program family that should be configurable in various different variants. It is a somewhat typical example of an embedded application software product line. The identified patterns and results are, however, equally relevant for the development of system software, which is our main field of research.

\subsection{Overview}

A weather station variant basically consists of one or more sensors to gain environmental information and one or more actors to process the gathered weather data. Figure 1 shows the possible variants of the weather station as a feature model. Note that the list of available sensors as well as actors is expected to grow in future versions, e.g. by additional sensors for wind direction and humidity. Not visible in the feature model, but nevertheless part of the product line definition, is that we have to distinguish between two kinds of actors:

generic actors are able to process / aggregate information of any set of sensors. Display and XMLProto are examples for generic actors. The XML representation of weather information, for instance, can easily and automatically be extended for additional sensors. 

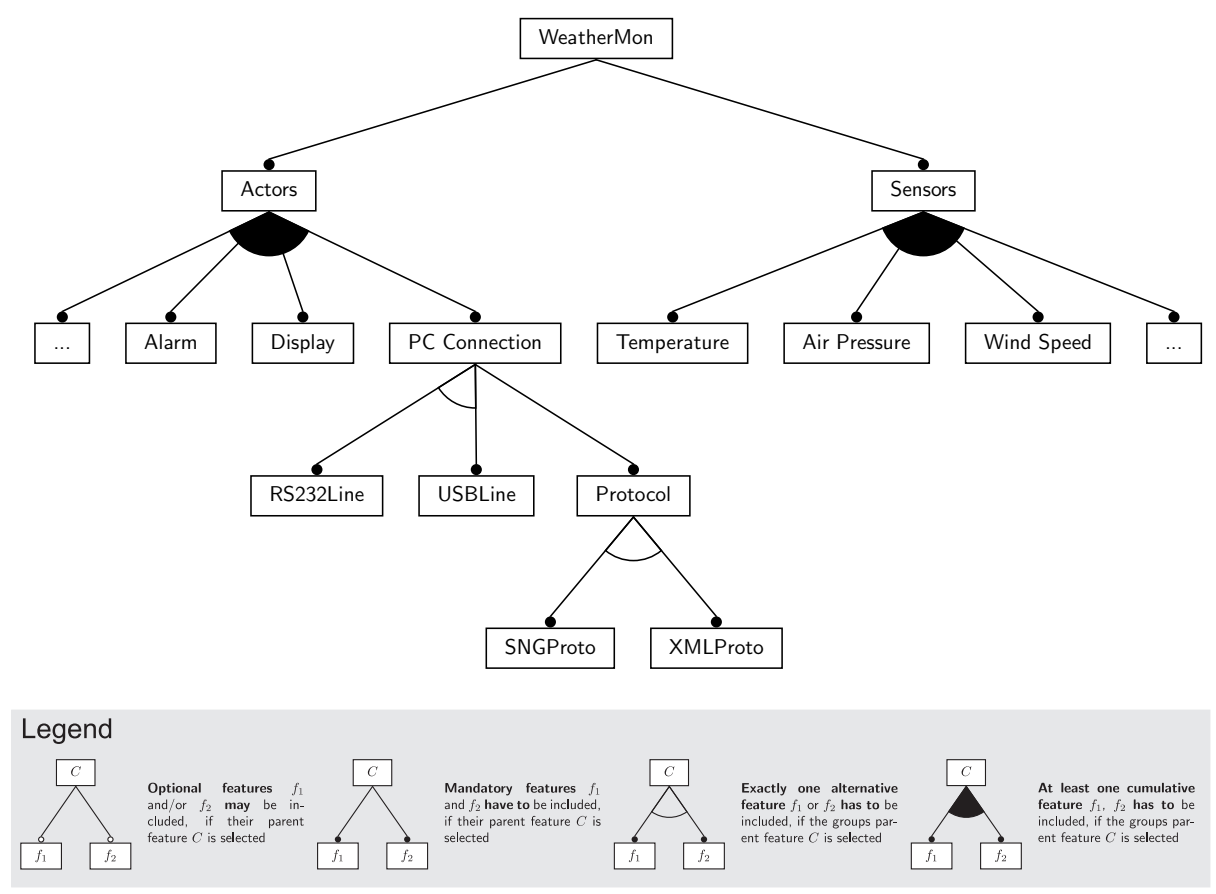

Fig. 1. Feature diagram of the embedded weather station product line. A WeatherMon variant consists of at least one sensor and one actor. Sensors gain information about the environment, such as Temperature, Air Pressure, or Wind Speed. Actors process sensor data: Weather information can be printed on a (LCD-) Display, monitored to raise an Alarm if values exceed some threshold, and passed to a PC over a PCConnection, which can be either an RS232Line or an USBLine, using either an XML-based data representation (XMLProto) or a proprietary format (SNGProto).

non-generic actors process / aggregate sensor data of some specific sensors only. SNGProto is an example for a specific actor. It implements a legacy binary data representation for compatibility with existing PC applications. This format encodes information of certain sensors only.

\subsection{Hardware Platform}

On the hardware side, sensors and actors of the weather station are connected to a small $\mu$-controller (Figure 2). The AVR series by Atmel is a typical $\mu$-controller product line. It is based on an 8 bit RISC core and offered with a broad variety of on-board equipment: 0-4 KB RAM, 1-128 KB program memory (flash), various busses and connectors $\left(\mathrm{I}^{2} \mathrm{C}\right.$, serial lines, A/D converters). Wholesale prices of the chip scale between 0.3 EUR and 7 EUR. They depend mostly on the on-board equipment, especially the amount of RAM and flash memory.

The goal is to reach a similar level of scalability in the software product line, so that hardware costs do scale with the amount of selected features. 


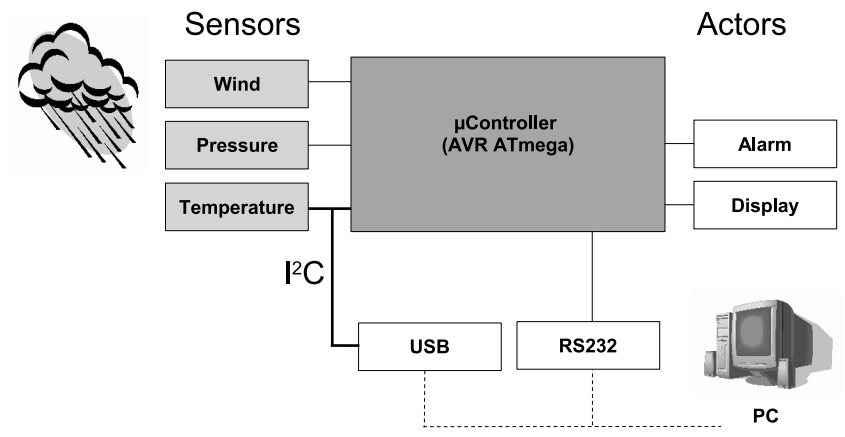

Fig. 2. Weather station hardware platform

\subsection{Configuration Process}

A weather station variant is configured by selecting features from the feature model. Conceptually, the feature model can be considered as the description of the configuration space, while a (valid) feature selection is a description of a concrete variant. In the implementation space, a family model provides a mapping from the abstract features to concrete implementation artifacts (such as .h and .cpp files) which implement the selected features. The feature selection is evaluated against the configurations space by a variant management tool[9], which copies the thereby defined set of implementation artifacts from the component repository into an output directory. The build process is then started in this directory to compile and link the configured system into the concrete variant (Figure 3).

The approach to map conceptual features by a family or platform model to concrete implementation artifacts is quite common the domain of embedded system software such as operating system product lines. ${ }^{1}$ The main advantages are flexibility and portability. By providing hardware-specific versions of the implementation space (family model and implementation components), the same conceptual OS model and configuration tool can be used to "generate" highly optimized variants for very different $\mu$-controller platforms and derivates.

The overall configurability (variability and granularity) of such product lines depend, however, on the (right-unique) mapping from implementation components to the features they implement, thus, a good separation of concerns. As a matter of fact, many features can not be mapped to single (C-language) artifacts, their implementation is intermingled within the implementation of other features. The result is scattered and tangled code, typically processed by an intra-artifact "second-order configuration" by means of the $\mathrm{C}$ preprocessor and conditional compilation. This hampers maintainability and evolvability of the implementation artifacts and thereby limits on the long term

\footnotetext{
${ }^{1}$ Examples are eCos[1], PURE[10], or many implementations of the OSEK system software standard used in automotive industry[2].
} 


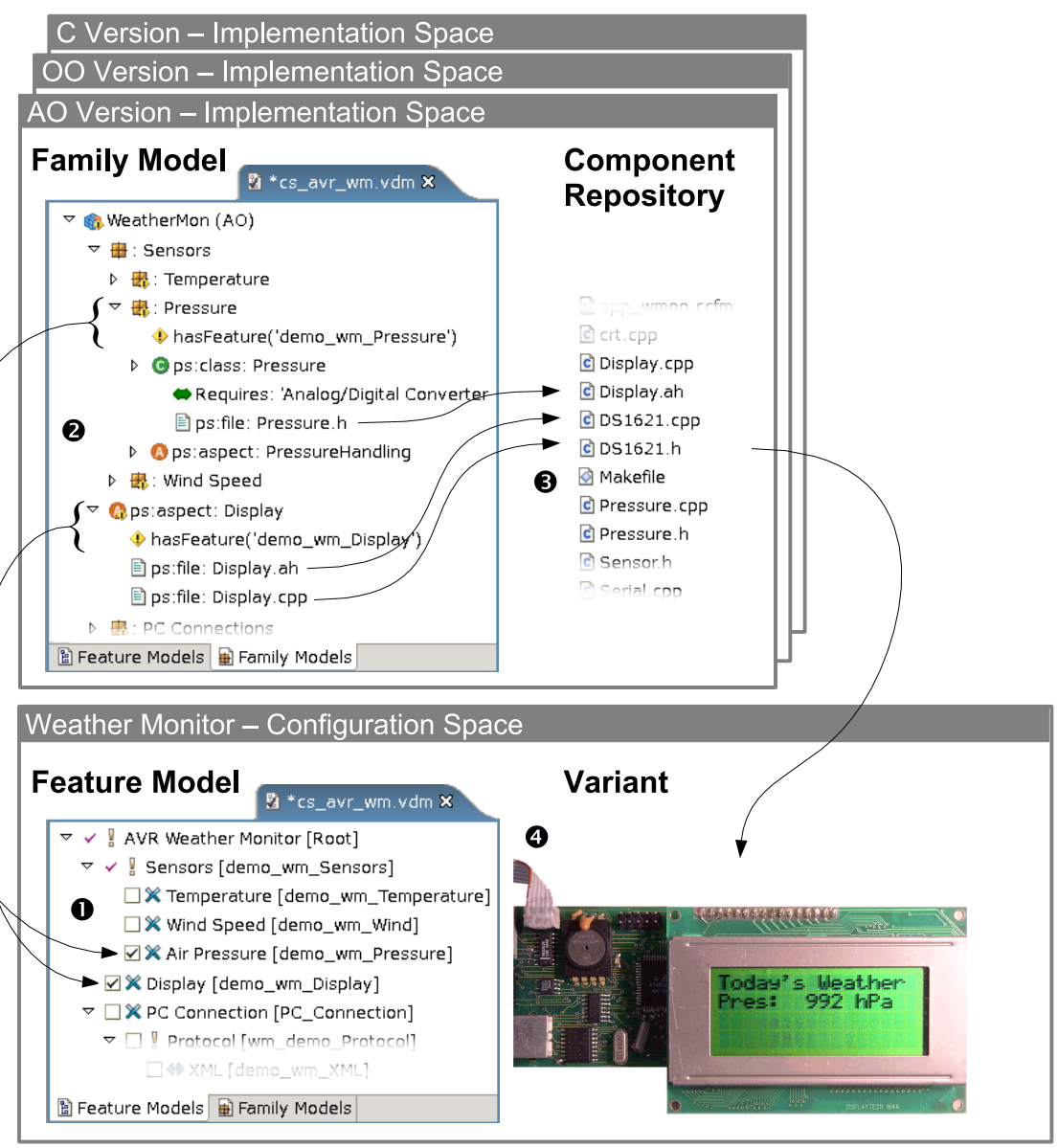

Fig. 3. Weather station configuration process. In the configuration space the user selects all wished features from the feature model (1). The implementation space consists of a family model and a component repository. The family model maps features to logical implementation components (2), which in turn are mapped to physical implementation files (3). The thereby determined set of implementation files for a concrete configuration is copied into the target directory, and finally compiled and linked into the actual weather station variant (4).

the extensibility of the whole product line. Hence, we strive for a better separation of concerns - as long as it does not lead to significant extra costs on the hardware side.

\section{Implementation}

In the following, we present and analyze two different implementations of the weather station product line that fulfill the goal of SoC. One implementation is OOP-based, while the other is AOP-based. The implementations were performed by two different, 
but equally experienced developers. Both have been (under the premise that SoC has to be achieved) carefully optimized with respect to resource requirements.

A third "traditional" C-based implementation is solely optimized for minimal resource consumption. Its only purpose is to provide the lower bounds of the resource consumption that can be reached in the different product line configurations.

The section is organized as follows: We start with a list of requirements in 3.1, which is followed by a description of the three implementations ( $\mathrm{C}$ in 3.2, $\mathrm{OO}$ in 3.3, and AO in 3.4). These descriptions are intended as a brief overview only. In particular, they do not motivate the chosen design of the $\mathrm{OO}$ and $\mathrm{AO}$ versions. Instead, all design decisions and idioms used to reach $\mathrm{SoC}$ in the $\mathrm{OO}$ and $\mathrm{AO}$ versions are discussed collectively (to set them in contrast with each other) in 3.5. The results are summarized in 3.6.

\subsection{Implementation Requirements}

Besides the functional features that have to be implemented, the additional requirements on the implementations can be summarized as resource-thriftiness (all versions) and separation of concerns ( $\mathrm{AO}$ and $\mathrm{OO}$ versions). This means in particular:

granularity Components should be fine-grained. Each implementation element should be either mandatory (such as the application main loop) or dedicated to a single feature only.

economy The use of expensive language features should be avoided as far as possible. For instance, a method should only be declared as virtual if polymorphic behavior of this particular method is required.

pluggability Changing the set of selected sensors and/or actors should not require modifications of any other part of the implementation. This basically means that sensor/actor implementations should be able to integrate themselves into the system.

extensibility The same should hold for new sensor or actor types, which may be available in a future version of the product line.

\subsection{Implementation of the $C$ Version}

Figure 4 shows the basic structure of the $\mathrm{C}$ version. Weather information is updated by the measure () function, which invokes - configured by conditional compilation - all existing sensors. For efficiency reasons, the actual weather data is passed as a global variable (not shown). Weather information is processed by the process() function, which invokes each actor (such as display_process ()) - again configured by means of conditional compilation. The ..._process () function of each actor retrieves weather information for all configured sensors it is interested in - once more using conditional compilation.

Due to the design goal to minimize the resource consumption, the $\mathrm{C}$ version does not fulfill our requirements on SoC (granularity, pluggability, extensability). By using global variables, conditional compilation and inlining of all functions that are referred only once, it offers, however, efficiency. 


\begin{tabular}{|c|c|c|c|}
\hline \multirow{3}{*}{ 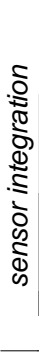 } & init_sensors (main.c) & measure (main.c) & display_process (display.h) \\
\hline & $\begin{array}{l}\text { inline void init_sensors }()\{ \\
\text { \#ifdef cfWM_WIND } \\
\text { wind_init(); } \\
\text { \#endif } \\
\text { \#ifdef cfWM_PRESSURE } \\
\text { pressure_init(); } \\
\text { \#endif }\end{array}$ & $\begin{array}{l}\text { inline void measure() } \\
\text { \#ifdef cfWM_WIND } \\
\text { wind_measure(); } \\
\text { \#endif } \\
\text { \#ifdef cfWM_PRESSURE } \\
\text { pressure_measure(); } \\
\text { \#endif }\end{array}$ & $\begin{array}{l}\text { inline void display_process }()\{ \\
\text { char val[ } 5 \text { ]; UInt8 line = } 1 \text {; } \\
\text { \#ifdef cfWM_WIND } \\
\text { wind_stringva7( val ); } \\
\text { display_print(1ine++, val, ..); } \\
\text { \#endif } \\
\text { \#ifdef cfWM_PRESSURE } \\
\text { pressure_stringval( val ); } \\
\text { display_print(1ine++, val, ...); } \\
\text { \#endif }\end{array}$ \\
\hline & \} & \} & \} \\
\hline & init sinks (main.c) & process (main c) & \multirow{3}{*}{ 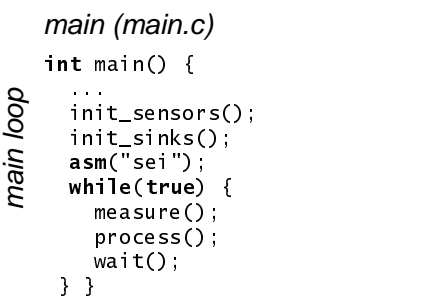 } \\
\hline 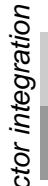 & $\begin{array}{l}\text { inline void init_sinks()\{ } \\
\text { \#ifdef cfWM_DISPLAY } \\
\text { display_init(); } \\
\text { \#endif } \\
\text { \#ifdef cfWM_PCCON_XML } \\
\text { XMLCon_init(); } \\
\text { \#endif }\end{array}$ & $\begin{array}{l}\text { in7ine void process() \{ } \\
\text { \#ifdef cfWM_DISPLAY } \\
\text { display_process(); } \\
\text { \#endif } \\
\text { \#ifdef cfWM_PCCON_XML } \\
\text { XMLCon_process(); } \\
\text { \#endif }\end{array}$ & \\
\hline & \} & \} & \\
\hline
\end{tabular}

Fig. 4. Static structure of / scattered code in the C version (excerpt)

\subsection{Implementation of the $\mathrm{OO}$ Version}

Figure 5 shows the class model of the OO version. Central elements are the Weather and Sink classes. Weather aggregates all sensors, which are registered at runtime by calling Weather: :registerSensor(). The Sink class aggregates all actors, respectively. Internally both, sensors and actors, are managed by chaining them into light-weight single-linked lists (ChainBase).

\section{Principle of Operation}

1. Weather information is acquired by calling Weather: :measure (), which in turn invokes the Sensor: : measure () method on every registered sensor to update the sensor data.

2. Weather information is processed by the Sink::process() method. Sink::process() first calls Actor::before_process() for each registered actor to initialize the processing.

3. Sensor information is passed to the actors by calling Actor: :process () for each registered sensor. Actors retrieve the actual sensor name, unit, and measured data (as character strings) by calling the respective Sensor methods.

4. At last, data processing is finalized by Sink::process() invoking Actor: : after_process () on each actor.

5. The whole process is repeated by the application main loop every second.

\subsection{Implementation of the AO Version}

The class/aspect model of the AO version is shown in Figure 6. Central elements are, again, the classes weather and Sink. Each sensor class is accompanied by a han- 


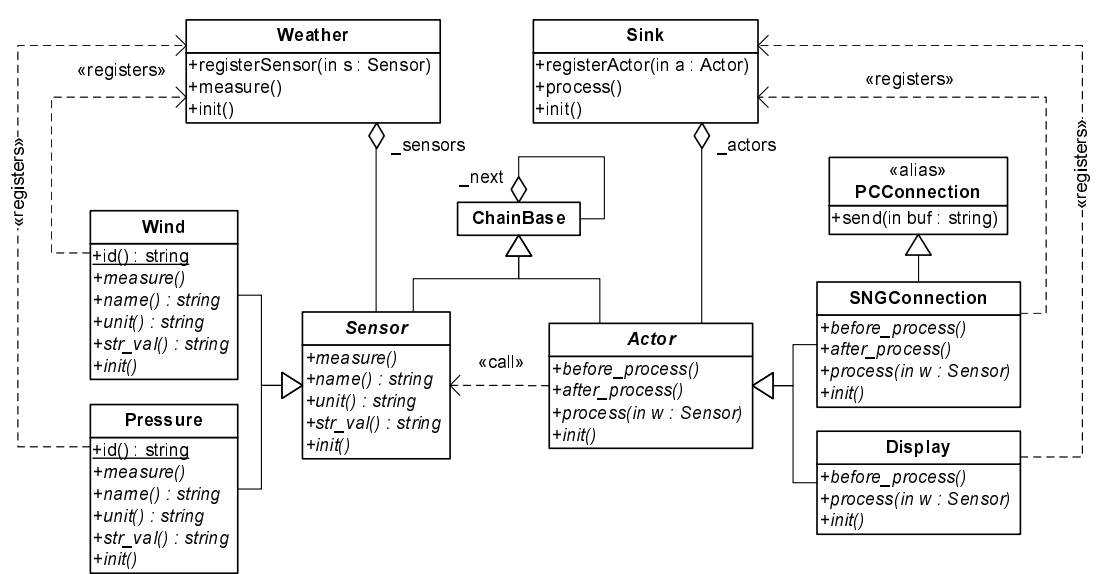

Fig. 5. Static structure of the OO version (excerpt with two sensors/actors, virtual functions are depicted in italics, static functions are underlined)

dling aspect, which performs the actual integration into Weather and Sink. A handling aspect performs two introductions: It aggregates the sensor as an instance variable into class weather and a sensor-specific (empty) process_data() method into class Sink. Additionally, it defines function execution advice for Weather: : measure () and Sink::process. Actors are implemented as aspects, which define execution advice for Sink: :process () (for initialization and finalization) and the sensor-introduced Sink: :process_data() methods (for the actual data processing).

\section{Principle of Operation}

1. Weather information is acquired by calling weather: :measure (), which is advised by the handling aspects of every sensor to call the sensor's measure () method.

2. Weather information is processed by the Sink::process() method. Sink::process() is before-advised by any actor that needs to initialize before processing.

3. Sink::process () is advised for each sensor to call the introduced sensor-specific process_data () method.

4. The sensor-specific process_data() method is advised by every actor that processes data of this sensor.

5. At last, data processing is finalized by Sink: : process () being after-advised for any actor that needs to finalize its processing.

6. The whole process is repeated by the application main loop every second.

\subsection{Used AOP and OOP Idioms}

To achieve the required level of SoC as well as pluggability and extensibility, both versions use approach-specific idioms and patterns in the design and implementation. In 


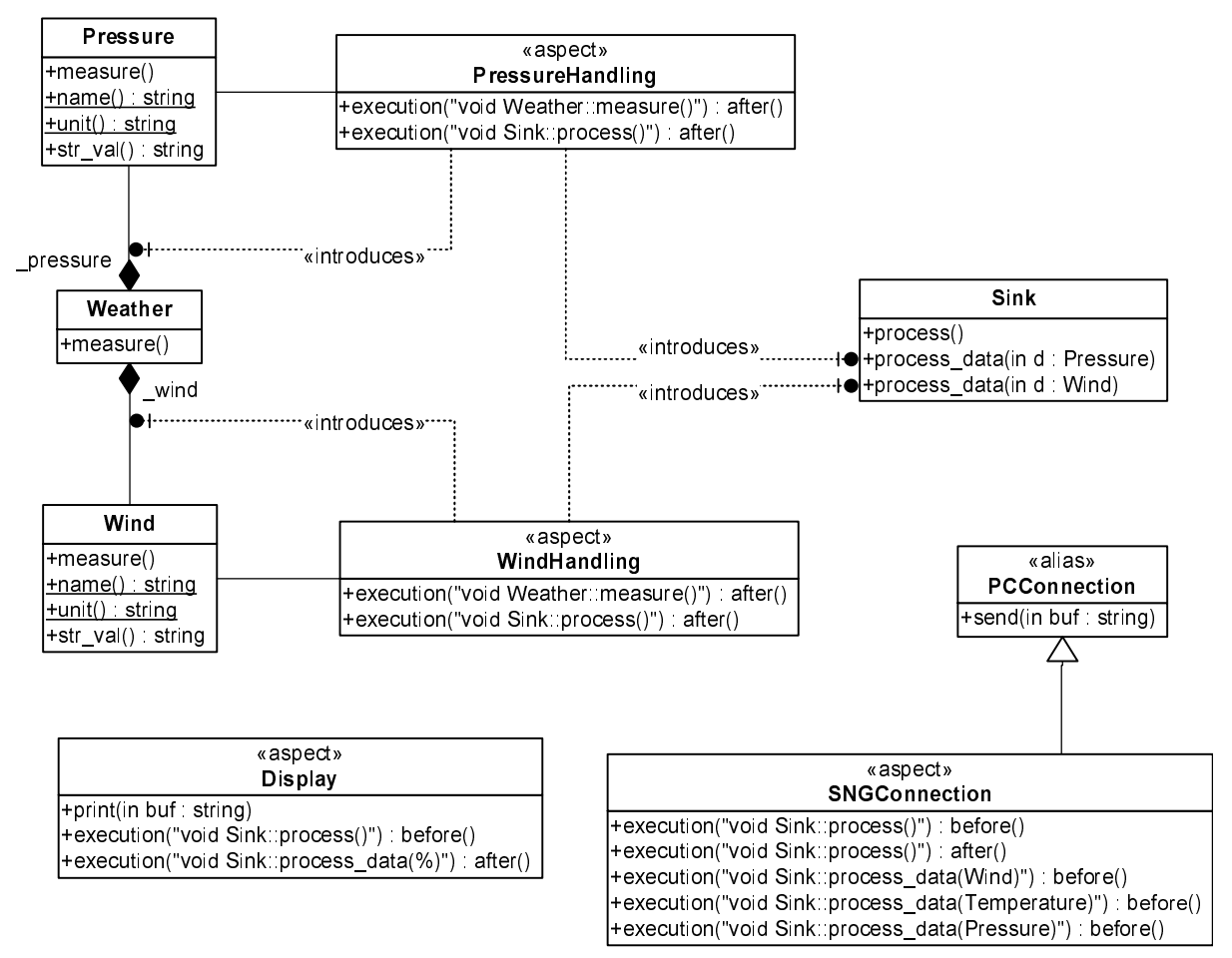

Fig. 6. Static structure of the AOP version (excerpt with two sensors/actors)

the following, we will analyze some interesting parts of the AOP and OOP implementations. The goal is to identify, compare, and discuss the idioms that have to be used to reach SoC. In particular, the following problems had to be solved:

\section{1) Working with configuration-dependent sensors/actors sets}

Both implementations have in common that the Weather/Sink classes are used to abstract from the configured sets of sensors and actors. These two abstractions are unavoidable, because the main () function, which performs the endless measurement and processing loop, should be configuration-independent and, thus, robust with respect to extensions. However, the implementation of the 1:n relationship of Weather/Sink and the concrete sensors and actors is completely different.

\section{Interface dependency between Weather/Sink and sensors/actors}

The Weather/Sink classes need to be able to invoke the measuring/processing of data independent of the actual sensor/actor types. Otherwise, pluggability and extensibility would be broken:

OO version: In the $\mathrm{OO}$ version, this is solved by common interfaces and late binding. Sensors have to inherit from the abstract class sensor, actors from the 
abstract class Actor, respectively. Weather/Sink invoke sensors/actors via these interfaces, thus depend on them. Sensor::measure(), Actor::before_process(), Actor: :after_process(), and Actor: :process() have to be declared as virtual functions:

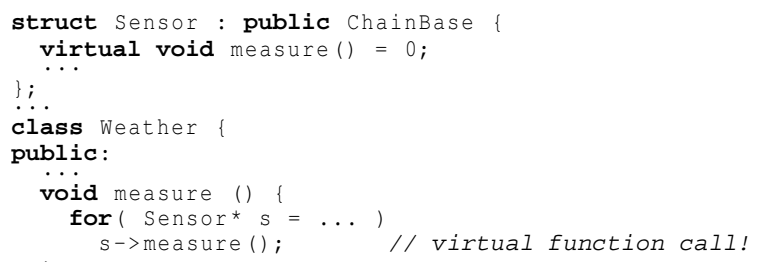

$A O$ version: In the $\mathrm{AO}$ version, the interface relationship is reverted. Weather and Sink do not depend on sensors/actors providing any specific interface. Sensors/actors are integrated by defining function execution advice for Weather: :measure () and Actor: : process ():

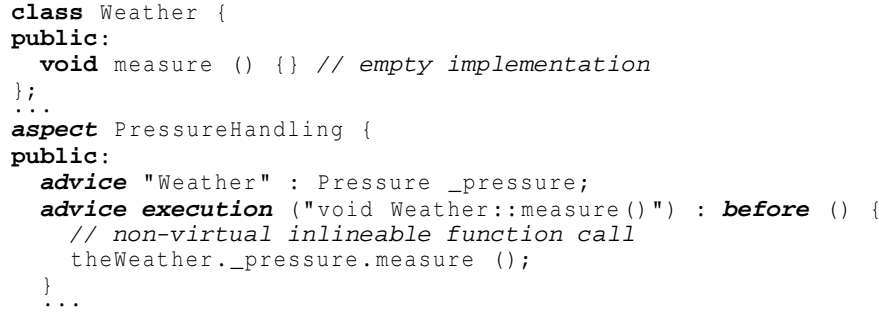

Potential cost drivers: In the OO version, four methods in two classes have to be declared and called as virtual functions. The AO version induces no costs, as all sensor/actor code can be inlined.

\section{Working with sets of sensors/actors}

Weather/Sink need to be independent of the actual number of sensors/actors configured into the system. There is a 1:n relationship between weather/Sink and sensors/actors:

OO version: In the $\mathrm{OO}$ version, this is solved by a simple publisher/subscriber mechanism. Sensors are chained into a linked list of "publishers", which is frequently iterated by Weather: : measure () to update the data of each sensor:

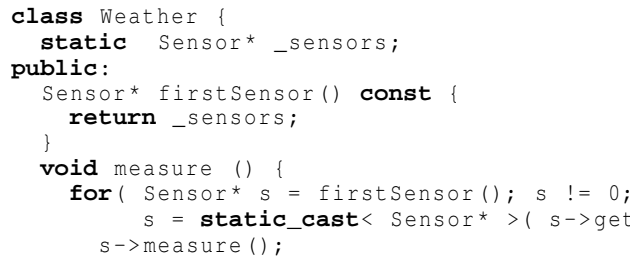


\}

Actors are similarly chained into a list of "subscribers" which are invoked if new sensor data is available. The sink class acts as "mediator" between sensors and actors. For the sake of memory efficiency, actors do not subscribe for single sensors, but are implicitly subscribed for the complete list.

$A O$ version: In the $\mathrm{AO}$ version, actors and sensors are implicitly chained at compiletime by multiple aspects defining advice for the same weather::measure() and Sink: :process () execution join points.

Potential cost drivers: In the $\mathrm{OO}$ version, Weather/Sink as well as sensors/actors need to carry an extra pointer for the chaining. Some additional code is required to iterate over the chain of sensors/actors. ${ }^{2}$

\section{Registration of sensors/actors}

This problem is closely related to the previous one. Sensors and actors need to be able to register themselves for the publisher/subscriber chain:

$O O$ version: In the $\mathrm{OO}$ version, registration is done at runtime by calling Weather: :registerSensor()/Sink: :registerActor(). Self-registration requires some $\mathrm{C}++$ trickery: Each sensor/actor is instantiated as global object. The registration is performed by the constructor, which is "automatically" triggered during system startup.

$A O$ version: In the $\mathrm{AO}$ version, no extra efforts are required for self-registration. The chaining of actors and sensors is implicitly performed at compile time by advice-code weaving. Basically, it is the presence of some aspect in the source tree that triggers the registration.

Potential cost drivers: In the $\mathrm{OO}$ version, the use of global object instances causes some overhead, as the compiler has to generate extra initialization code and puts a reference to this code in a special linker section. A system startup function has to be provided that iterates over this section and invokes the constructors of all global instances.

\section{2) Implementation of generic actors}

Generic actors process sensor data from any sensor type. For example, the Display actor should print the measured values of any configured sensor, regardless of the current configuration or future extensions with new sensor types. However, this leads to an interface dependency between actors and sensors, because (at least) the value has to be obtained in a generic way:

\footnotetext{
2 The firstSensor() and getNext () operations are, however, inlined as they perform just a pointer lookup.
} 
OO version: In the $\mathrm{OO}$ version, this is again solved by interfaces and late binding. To enable actors to retrieve sensor information from any sensor, the sensor interface has to be extended by three additional virtual functions: Sensor: : name (), Sensor: : unit (), and Sensor: :str_val ():

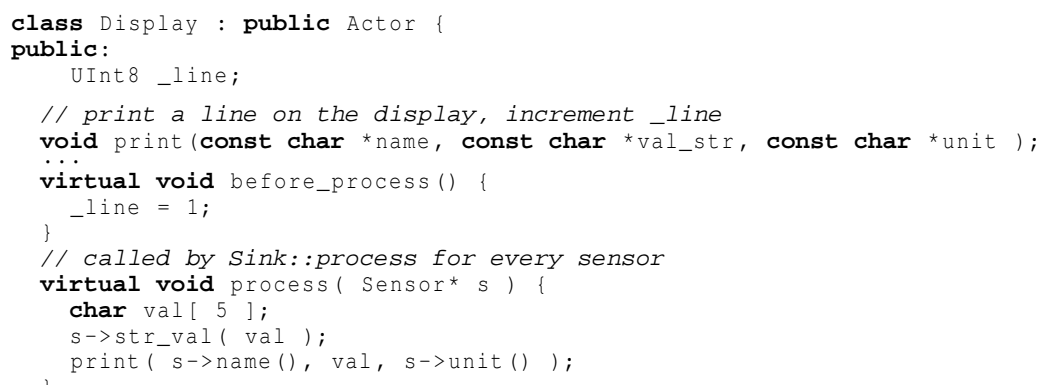

$A O$ version: In the $\mathrm{AO}$ version, this is solved by using the AspectC++ concept of generic advice [32,35]. For every sensor Mysensor, the corresponding MySensorHandling aspect introduces an empty sensor-specific process_data(const MySensor\&) method into class Sink and gives advice to Sink: :process () to invoke the introduced method:

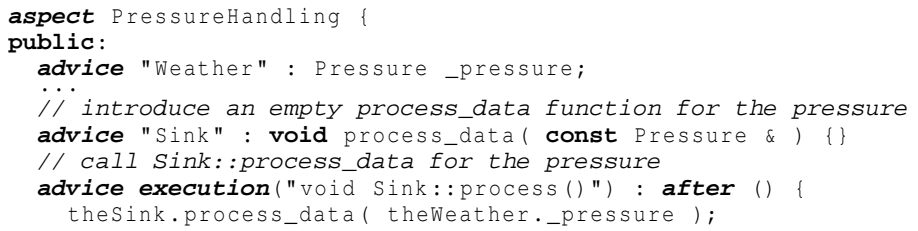

An actor gives generic advice that matches every (overloaded) Sink: :process_data() function. To be independent of the actual sensor type, the advice body uses the AspectC++ join point API to retrieve a typed reference to the sensor instance:

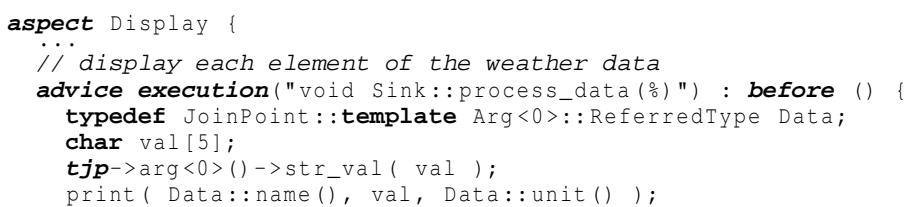

AspectC++ instantiates advice bodies per join point. Therefore, the calls to the actual sensor's str_val (), name (), and unit () methods can be bound at compile-time. As an additional optimization, name () and unit () are implemented as static (class) functions.

Potential cost drivers: In the $\mathrm{OO}$ version, three additional virtual functions are required in the sensor classes, as well as additional virtual function calls in the actor classes. In 
the AO version, the join point API has to be used for a uniform and type-safe access to the sensor instance, which induces some overhead. Furthermore, the advice body of a generic actor is instantiated once per sensor, which may lead to code bloating effects.

\section{3) Implementation of non-generic actors}

Non-generic actors process data of some sensors only. The legacy SNG protocol, for instance, encodes weather data in a record of wind speed, temperature and air pressure. It exposes the actual data using sensor-specific interfaces. The record may be sparse, meaning that a specific sensor, such as Temperature, may or may not be present in the actual system configuration.

$O O$ version: In the $\mathrm{OO}$ version, a non-generic actor filters the sensors it is interested in by runtime type checks in the process () method. Each passed sensor is tested against the handled sensor types. If the runtime type matches with a handled sensor, a downcast is performed to get access to the sensor-specific interface:

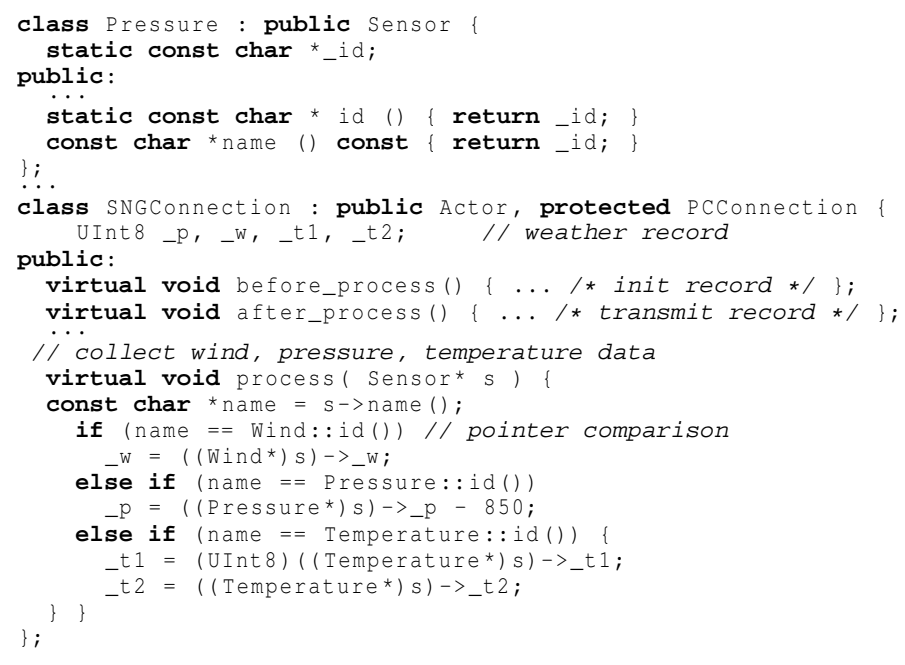

The idiom commonly used for runtime type checks in $\mathrm{C}++$ is the dynamic_cast operator, which is part of the $\mathrm{C}++$ runtime type interface (RTTI). RTTI is, however, quite expensive, as it requires additional runtime support and leads to some extra overhead in every class that contains virtual functions. To avoid this overhead, our implementation uses a "home-grown" dynamic type-check mechanism: The test is performed by comparing the string address returned by the (late-bound) Sensor: : name () method with the address of the name string stored in the concrete class ${ }^{3}$, which is also returned by the static Sensor: :id() method. The expensive C++ RTTI mechanism has been disabled.

Normally, dynamic type checks are considered harmful, because of a lack of extensibility and the accumulated costs of type checks, which sometimes outweigh the

\footnotetext{
${ }^{3}$ This basically reduces the overhead of a runtime type test to a virtual function call and a pointer comparison. As the storage for the name string has to be provided anyway, this mechanism also induces no extra overhead in the sensor classes.
} 
costs of a single virtual function call. However, in our case a non-generic actor shall be implemented. Therefore, extensibility is not an issue and the overhead of our type check implementation is acceptable. At the same time, alternative designs such a visitor [24] fail, because a Visitor interface would have to list a visitSensor() method for every sensor type. However, the set of sensors is configurable and should nowhere be hard-wired.

$A O$ version: In the $\mathrm{AO}$ version, binding an actor to selected sensors only is realized by giving advice for specific Sink: :process_data () methods only instead of using generic advice:

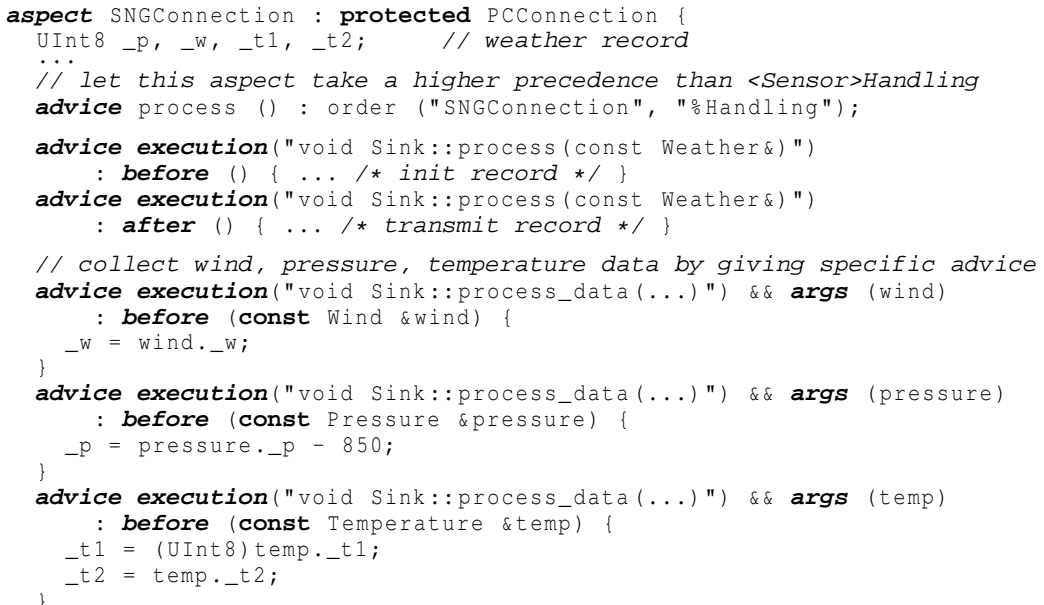

Potential cost drivers: Runtime type checks in the OO version induce nevertheless some overhead. In the AO version, some overhead is induced by the args () pointcut function, which is used here to get the actual sensor instance.

\subsection{Implementation Summary}

Both, the OOP as well as the AOP implementation of the embedded weather station product line provide good SoC. In particular, the implementation requirements described in section 3.1 are met by both versions:

granularity is achieved by the $\mathrm{OO}$ as well as the $\mathrm{AO}$ version. Each implementation component is either mandatory (such as the Weather and Sink classes), or dedicated to a single feature only.

economy is achieved as far as possible. In the OO version, only methods that have to be available via a generic interface are declared as virtual. RTTI is not used, as the required runtime type checks can be implemented with less overhead. In the AO version, join point-specific context information is used only sparingly. 
pluggability is achieved as well. In both versions, no component has to be adapted if the set of selected sensors/actors is changed. Sensors and actors basically integrate themselves, if their implementation component is present in the configured source tree. The $\mathrm{OO}$ version uses global instance construction for this purpose. In the AO version, the integration is performed by advice.

extensibility is also achieved. In the OO version, new sensor/actor types just need to implement the common Sensor/Actor interface. In the AO version, new sensor/actor types just need to provide some aspect that performs the integration into Weather/Sink.

Overall, the $\mathrm{AO}$ and $\mathrm{OO}$ versions are equipollent from the SoC viewpoint. We identified, however, noticeable more potential cost drivers in the OO version than the AO version. Especially virtual functions were unavoidable in many places to realize loose coupling and genericity of components. In the next section, we analyze how this affects scalability and memory demands of the product line.

\section{Cost Analysis}

In this section, we analyze scalability and memory requirements of the embedded weather station product line. For this purpose, several configurations of the weather station were generated as $\mathrm{AO}, \mathrm{OO}$, and $\mathrm{C}$ variants. ${ }^{4}$ For each variant, we measured:

- static memory demands, which are determined by the amount of generated machine code (text), static initialized data (data) and static non-initialized data (bss).

- dynamic memory demands, which are determined by the maximum stack space used by the running application (stack). ${ }^{5}$

- the runtime of a complete measure/process-cycle.

On the actual hardware, text occupies flash memory space. Data occupies flash memory and RAM space, as it is writable at runtime and therefore has to be copied from flash into RAM during system startup. Bss and stack occupy RAM space only.

\subsection{Measurement Methods}

Static memory demands (text, data, bss) could easily be retrieved directly from the linker map file. Dynamic memory demands (stack) and runtime had to be measured in the running targets:

\footnotetext{
${ }^{4}$ All variants were compiled with avr-g++ (GCC) 3.4.1 using -Wall -fno-rtti -Os -fno-exceptions -fomit-frame-pointer -ffunction-sections optimization flags. $\mathrm{AO}$ variants were woven with ac++ 0.9.3.

5 The weather station software uses no heap, which otherwise would also contribute to dynamic memory demands.
} 


\section{Measuring Stack Utilization}

For simple programs it is possible to determine the stack utilization off-line and byteexact by static analysis of the machine code. Unfortunately in our case the program execution graph is not predictable due to the use of interrupts and late bound functions. Therefore we used runtime monitoring of the stack as a pragmatic alternative. A common technique for runtime stack monitoring is to initialize the entire stack space with some specific magic pattern during system startup. The maximum amount of stack used can then be measured at any time by searching (from bottom of stack) for the first byte where the pattern has been overwritten.

In the weather station variants, this technique was used to implement stack measurement as an additional sensor type. Understanding stack measurement as just another sensor had some nice advantages. Because of the achieved pluggability (in the $\mathrm{AO}$ and $\mathrm{OO}$ versions) it was very easy to apply stack measurement to any weather station configuration. Of course, some extra care had to be taken to ensure that the maximum stack utilization is not caused by the stack measurement sensor itself. For this reason, the stack measurement implementation uses only global variables which do not occupy stack space. By analyzing the generated machine code we ensured that the stack utilization of the stack sensor methods is minimal among all sensors. As all sensor methods are invoked from the same call depth level and at least one "real" sensor besides stack measurement is used in a weather station configuration, it can thereby be guaranteed that stack measurement itself does not tamper the maximum stack utilization. In the actual targets, the thereby acquired maximum stack utilization remained stable after a short startup time and could be read from one of the attached generic actors.

\section{Measuring Runtime \\ Runtime measuring was not implemented as another sensor type, as it had been too difficult to distinguish the runtime taken by the sensor processing itself from the run- time of the target to measure. Instead, we used a less invasive approach that could be implemented with just two additional assembler statements: In the application main loop, a digital I/O port of the AVR $\mu$-controller is set to high before the call to Weather: :measure () and reset to low after the return of Sink: :process (). The result is a rectangular signal on this port, which was recorded and analyzed with a storage oscilloscope $^{6}$. A high period in the signal represents the runtime taken by a complete Weather: :measure() / Sink: :process() cycle. After a short startup time, period and phase of the signal remained stable and the length of the high phase could be measured.}

\subsection{Overall Scalability of the Product Line}

As the graphs in Figure 7 show, the resulting RAM/flash demands of the weather station software do scale quite well with the amount of selected features. The "Barometer" configuration (P+Display), consisting of just an air pressure sensor and an LCD display, induces significantly smaller memory demands than the "Deluxe-PC" configuration (TWP+Serial+XML+Display) which bundles three sensors, a LCD display, and

\footnotetext{
${ }^{6}$ Tektronix TDS 2012, 100MHz resolution
} 

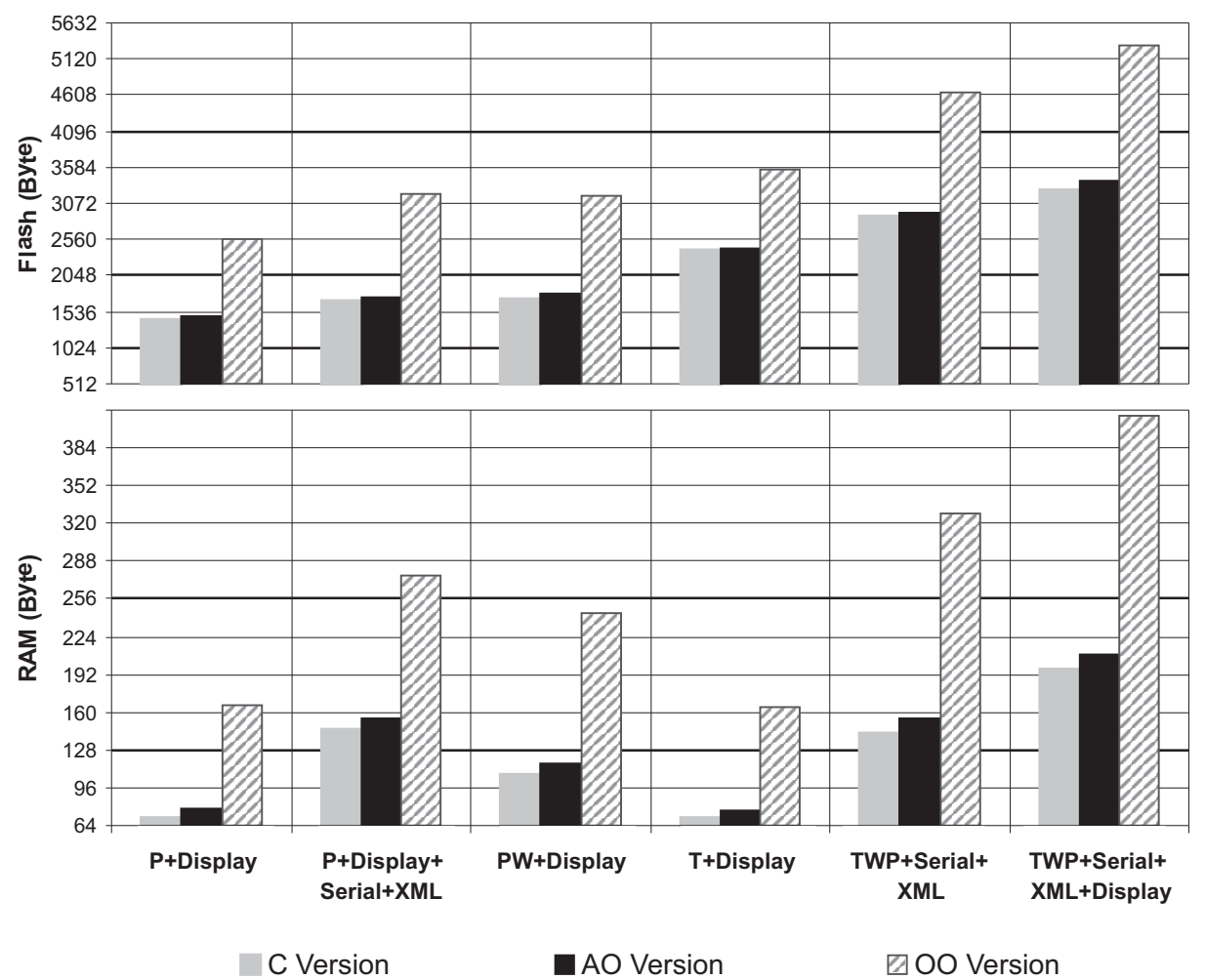

Fig. 7. Footprint comparison (RAM, flash) for different configurations

a XML-based PC connection over a serial interface. The memory requirements of the other examined configurations are in between. The noticeably high amount of flash memory required by the "Thermometer" configuration ( $\mathrm{T}+$ Display) can be explained by the fact that this sensor is connected via the $\mathrm{I}^{2} \mathrm{C}$ bus to the $\mu$-controller (see Figure 2). To drive this bus, additional driver code is required that has not to be included for other sensors.

Overall, all three versions meet the goal of scalability, which is an indicator for achieved granularity. In every case, however, the OOP version requires significantly more memory space than its AOP counterpart which comes very close to the $\mathrm{C}$ version. Depending on the configuration, the required amount of RAM is up to $138 \%$ higher in the $\mathrm{OO}$ version, while the $\mathrm{AO}$ version takes only an extra of $10 \%$ at maximum (up to 13 byte)-both compared to the $\mathrm{C}$-based version that does not provide SoC. The amount of flash memory is up to $91 \%$ higher in the OO version, but only $4 \%$ at maximum in the AO version. The net difference between using AOP and OOP has to be considered as even higher, as both versions of each configuration are linked with the same (configuration- 


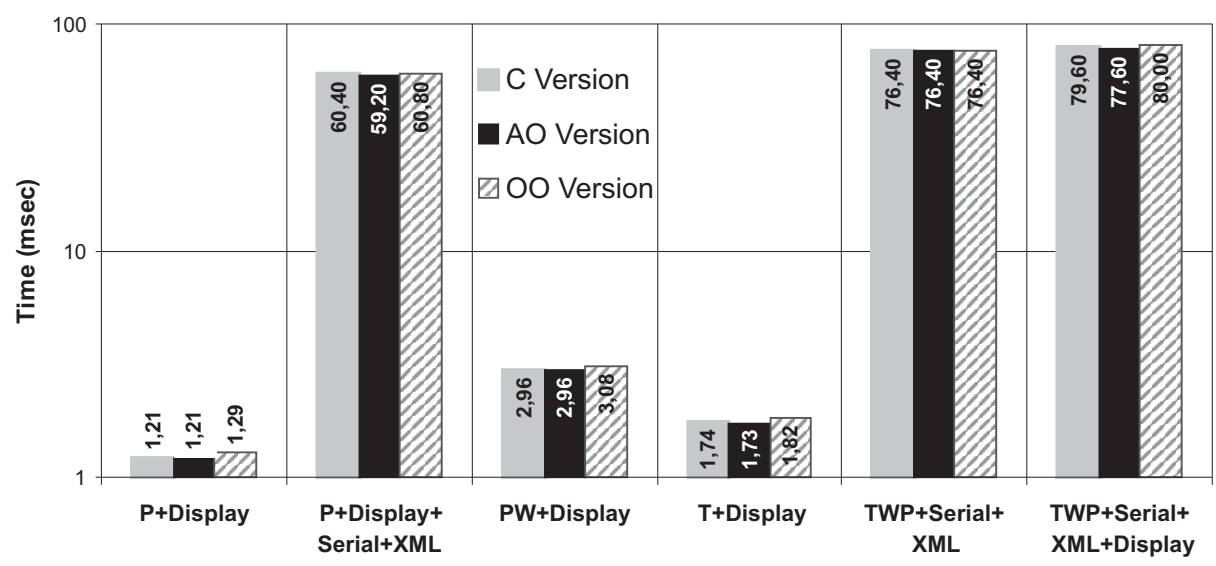

Fig. 8. Footprint comparison (time taken by a Weather: : measure () / Sink: : process () cycle) for different configurations.

dependent) set of device drivers whose memory requirements are included in these numbers. ${ }^{7}$

The runtime of all three versions is almost the same in all configurations (see Figure 8). It is mostly dominated by hardware and driver-related costs.

\subsection{Memory Requirements in Detail}

Table 1 breaks down the required overall amount of RAM and flash memory into their origins. It is evident, that the $\mathrm{OO}$ variants induce especially higher static memory demands. The text sections of the OO variants are up to $78 \%$, the data sections up to $284 \%$ bigger than in the $\mathrm{AO}$ variants. The following cost drivers can be mainly accounted for this:

virtual functions are the main source of additional code and data, as they induce overhead on both, the caller and the callee side. On the caller side, each call to a virtual function requires, compared to advice code inlining, at least 16 additional byte in the machine code. On the callee side, virtual function tables have to be provided ( 4 byte +2 byte per entry, data), object instances need to carry a pointer to the virtual function table ( 2 byte per instance), and constructors to initialize the vtable pointer of an instance have to be generated (at least 24 byte per class, text). In the "Barometer" configuration (P+Display), for instance, 52 byte of the data section are occupied solely by virtual function tables. ${ }^{8}$

Regarding code size, the situation may become even worse in larger projects: Due

\footnotetext{
${ }^{7}$ As parts of the driver code are implemented as inline functions, it is not possible to differentiate between driver-induced and application-induced code here.

${ }^{8}$ The AVR RISC core uses a Harvard architecture, thus vtables can not be placed in the text section.
} 
Table 1. Memory usage and runtime of the $\mathrm{AO}$ and $\mathrm{OO}$ versions for different configurations

\begin{tabular}{|c|c|c|c|c|c|c|c|c|}
\hline \multicolumn{2}{|l|}{ Configuration } & text & data & bss & stack & flash & ram & time \\
\hline \multirow[t]{3}{*}{ P+Display } & $\mathbf{c}$ & 1392 & 30 & 7 & 34 & 1422 & 71 & 1.21 \\
\hline & AO & 1430 & 30 & 10 & 38 & 1460 & 78 & 1.21 \\
\hline & oo & 2460 & 100 & 22 & 44 & 2560 & 166 & 1.29 \\
\hline \multirow{3}{*}{$\begin{array}{l}\text { P+Display+ } \\
\text { Serial+XML }\end{array}$} & $\mathrm{C}$ & 1578 & 104 & 7 & 34 & 1682 & 145 & 60.40 \\
\hline & AO & 1622 & 104 & 12 & 38 & 1726 & 154 & 59.20 \\
\hline & oo & 3008 & 206 & 26 & 44 & 3214 & 276 & 60.80 \\
\hline \multirow[t]{3}{*}{ PW+Display } & $\mathrm{c}$ & 1686 & 38 & 14 & 55 & 1724 & 107 & 2.96 \\
\hline & AO & 1748 & 38 & 18 & 61 & 1786 & 117 & 2.96 \\
\hline & oo & 3020 & 146 & 33 & 65 & 3166 & 244 & 3.08 \\
\hline \multirow[t]{3}{*}{ T+Display } & $\mathrm{c}$ & 2378 & 28 & 8 & 34 & 2406 & 70 & 1.74 \\
\hline & AO & 2416 & 28 & 11 & 38 & 2444 & 77 & 1.73 \\
\hline & oo & 3464 & 98 & 23 & 44 & 3562 & 165 & 1.82 \\
\hline \multirow{3}{*}{$\begin{array}{l}\text { TWP+Serial+ } \\
\text { XML }\end{array}$} & $\mathrm{c}$ & 2804 & 90 & 17 & 35 & 2894 & 142 & 76.40 \\
\hline & AO & 2858 & 90 & 23 & 41 & 2948 & 154 & 76.40 \\
\hline & oo & 4388 & 248 & 39 & 41 & 4636 & 328 & 76.40 \\
\hline \multirow{3}{*}{$\begin{array}{l}\text { TWP+Serial+ } \\
\text { XML+Display }\end{array}$} & & 3148 & 122 & 17 & 57 & 3270 & 196 & 79.60 \\
\hline & AO & 3262 & 122 & 24 & 63 & 3384 & 209 & 77.60 \\
\hline & oo & 5008 & 300 & 44 & 67 & 5308 & 411 & 80.00 \\
\hline
\end{tabular}

to late binding, the bodies of virtual functions are never removed from the final image by means of function-level linking. Thus, "dead" function code that is never called at runtime becomes nevertheless part of the text section.

dynamic data structures are another source of additional overhead. The chaining of actors and sensors induces 8 additional data byte in the "Barometer" configuration, plus some extra code to access and iterate over the lists.

global instance construction causes some more "hidden" code to be generated. For each translation unit that defines one or more global objects, the compiler has to generate a specific initialization-and-destruction function ( 88 bytes, text). Pointers to these functions are additionally stored in the data section.

Regarding dynamic memory usage (stack), the differences between the $\mathrm{AO}$ and $\mathrm{OO}$ version are less significant. The $\mathrm{OO}$ variants need a few byte (up to $16 \%$ ) more stack space than the related $\mathrm{AO}$ variants. This seems surprising at first, given that virtual function calls can not be inlined, therefore lead to a higher call depth and, thus, higher stack utilization. Part of this effect can be explained by the fact that the AOP version requires some additional stack space as well, namely by context-binding pointcut functions and the join point API (2-4 byte). The main reason is, however, that the maximum virtual function call depth is with 2 levels quite low. As the AVR architecture provides 32 general-purpose registers, which are also used for passing function parameters, it can furthermore be considered as quite "stack-friendly". On other CPU architectures (such as Intel), the differences between $\mathrm{AO}$ and $\mathrm{OO}$ based solutions would be more significant. 


\subsection{Runtime Requirements in Detail}

Table 1 also lists the measured runtimes in detail. In all configurations that support a serial connection the measurement/processing cycle time is mainly dominated by the underlying serial device driver. Here the costs are almost the same. In all other configurations the performance of the $\mathrm{AO}$ version and our highly efficient $\mathrm{C}$ implementation are the same. The runtime overhead of the $\mathrm{OO}$ version is between $4 \%$ and $6.6 \%$. It can be explained with the numerous virtual function calls on the application level.

\subsection{Cost Analysis Summary}

Both, the $\mathrm{AO}$ and $\mathrm{OO}$ version of our product line, scale quite well. The runtime differences are small and only of minor importance in this domain. The OO version, however, induces dramatically higher memory requirements. Given a hardware product line like the AVR ATmega series, these differences can directly be mapped to the required $\mu$-controller features. As Figure 9 shows, the hardware costs of the C-based and AObased product line would be exactly the same in all configurations. Using AO versus OO leads even for our small example product line to significant differences regarding hardware costs. This effect would probably be even higher for larger product lines.

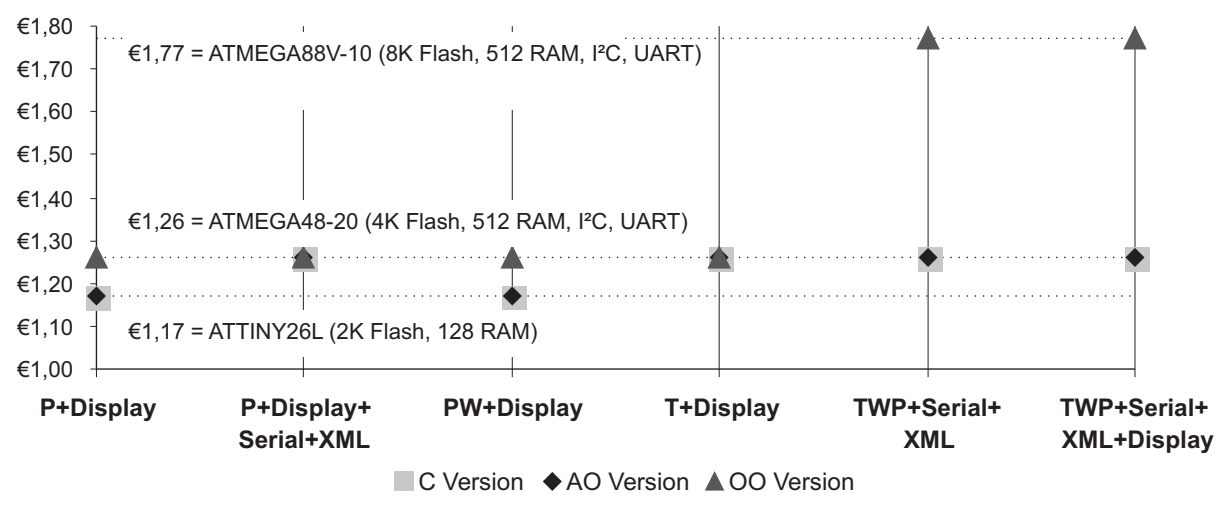

Fig. 9. Hardware scalability (required $\mu$-controller variant) of the $\mathrm{AO}$ and $\mathrm{OO}$ versions for different configurations (wholesale prices: Digi-Key Corporation, Product Catalogue, Summer 2005)

\section{Discussion}

As pointed out in the previous sections, the AOP-based version of the weather station product line combines good SoC with high memory efficiency. In the following we discuss the principal reasons for this effect, the potential disadvantages, and to what degree our results are applicable to (system software) product lines in general and achievable with other AOP approaches. 


\subsection{General Advantages of the Approach}

To ensure variability and granularity in product lines, components need to be implemented in a fine-grained and loosely coupled way. In our case study, we identified three fundamental concepts that are required to reach this: Abstraction from concrete components (interfaces), abstraction from component sets (1:n relationships), and abstraction from component integration (self-pluggability). The major disadvantage of OOP is that the features it provides to reach these goals are basically runtime concepts, while with AOP (in the case of static weaving) it is possible to use compile-time concepts:

abstraction from concrete components requires to use interfaces and runtime binding in OOP (thus, virtual functions in $\mathrm{C}++$ ), while in AOP advice code is woven at compile-time to the join points (and in AspectC++ is even inlined).

abstraction from component sets requires to use some kind of runtime data structures in OOP, such as linked lists, while in AOP multiple advice can be woven at the same join point. Moreover, by means of aspect ordering, it is even possible to define a context-dependent invocation order.

abstraction from component integration is generally difficult to achieve in most OOP languages. In $\mathrm{C}++$ it requires some constructor trickery to perform the integration at runtime. In AOP, Advice is per se an integration mechanism and resolved at compile-time. Moreover, aspects provide means for context-sensitive integration: Giving advice for a specific join point can be understood as a weak reference to another component, as the advice is silently ignored if the join point does not exist.

\subsection{Compiler Issues}

As pointed out in the analysis, much of the extra overhead of the $\mathrm{OO}$ version is directly or indirectly induced by the costs of late binding, even though the extra flexibility at runtime is not required in every case. A lot of work has been conducted to reduce the costs of $\mathrm{C}++$ virtual functions $[3,8]$ and late binding in general, e.g. by static whole program optimization [18]. This gives rise to the question, if and to what extend the measured $\mathrm{OO}$ overhead could be prevented by better compilers.

Especially in the context of the highly complex and non-academic programming languages $\mathrm{C}$ and $\mathrm{C}++$, which are the state of the art in system software and embedded software development, the implementation of optimizations based on static whole program analysis is extremely difficult. Although many of the aforementioned algorithms and techniques were proposed at least 10 years ago, today's commercial compilers have not incorporated these innovations yet. The main reasons are their complexity and existing de-facto standards like object file formats and ABIs. ${ }^{9}$ Therefore, we pragmatically advocate for using $\mathrm{OO}$ and $\mathrm{AO}$ mechanisms in combination in order to get the best out of both worlds. While $\mathrm{OO}$ is well suited for applications that actually need dynamism, static advice weaving is an interesting alternative that avoids the $\mathrm{OO}$ overhead from the beginning if dynamism is not required.

\footnotetext{
${ }^{9} \mathrm{ABI}=$ Application Binary Interface
} 


\subsection{Applicability to System Software}

Compared to application software product lines, system software product lines have to fulfill even higher demands on configurability and resource-efficiency. With respect to efficiency, the above mentioned abstractions are particularly useful in system software:

efficient callbacks by advice code inlining. System software usually has to provide means to bind user-defined code to (asynchronous) system events, such as hardware interrupts in an operating system. To prevent uses-dependencies[41] of the system code to driver/application code, a callback or upcall mechanism via functionpointers has to be provided. This leads to extra overhead and higher latencies at runtime. As aspects reverse such dependencies[14], they provide means to break uses-dependencies without this extra overhead.

handler chains by multiple advice. The implementation of handler chains can be implemented by the same mechanism as single callbacks. Due to advice code inlining, this induces furthermore no additional overhead.

configurable initialization order by aspect precedences. In system software it is often necessary to initialize components and subsystems in some well-defined (partial) order during system startup. The order is determined by componentinterdependencies, however, not necessarily fixed in the case of configurable product lines. It may well depend on the actual configuration. By aspect precedences (given as order advice in AspectC++), aspects provide perfect means to solve this problem at compile-time.

\subsection{Potential Disadvantages of the Approach}

The advantages regarding resource efficiency are mainly caused by binding and inlining component code at compile-time that is bound at runtime in OOP. This may, however, also lead to limitations of the approach:

compile-time fixation limits the approach to static configuration of product lines. In domains, where the set of selected features can arbitrary change at runtime, additional support has to be provided e.g. by a dynamic aspect weaver. As most dynamic weaving approaches induce inevitable overhead[27], it is questionable if in such cases AOP still out-weights the overhead of OOP. However, in most cases only some features can be expected to change at runtime. A tailorable low-cost weaving infrastructure, which provides means to configure per-feature if static or dynamic weaving should be used [45], may be a promising solution for such cases, as this enables developers to optimize the trade-off between dynamism and resource overhead at configuration time.

code bloating due to advice code inlining. This occurs if functionality given by advice is invoked from (and therefore woven to) many different join points. While this situation has to be considered for highly cross-cutting concerns such as tracing, component composition usually takes place at few well-known join points only. In any case, however, the programmer is able to prevent this effect by simply moving the advice body into a non-inlined function that is invoked from the advice code. 


\subsection{Applicability to Other AOP Approaches}

Our results show, that in the case of $\mathrm{C}++$ and AspectC++, using AOP instead of OOP may lead to significant saving effects in terms of memory and hardware costs. This gives rise to the question, if similar results can be reached with other languages and/or AOP approaches as well. This is clearly a huge field for further research and can not be answered in this paper. We think, however, that the following points are of particular importance:

weaver runtime support may lead to some unavoidable base overhead that limits the reachable amount of memory savings. This is clearly an issue for the domain of embedded systems. Experiments conducted with AspectJ on Java2 Micro Edition, for instance, have shown that the AspectJ runtime induces an extra $35 \mathrm{~KB}$ overhead[49]. AOP approaches that do not require runtime library support, are probably better suited for the goal of resource-thriftiness. Besides AspectC++, XWeaver[44] and AspectC[12], for instance, may be promising candidates.

language capabilities of the host language have a high influence on the applicability of the approach. As the resource savings are mainly caused by using compile-time instead of runtime concepts, the host language has to support such concepts. On the one hand, it is probably difficult to get "rid of the OOP overhead" in languages that have been particularly designed as object-oriented languages, such as Java or $\mathrm{C \#}$. On the other hand, languages such as $\mathrm{C}$ may not be sufficiently expressive with respect to (compile-time) genericity to take full advantage of the approach.

\subsection{Design Issues}

The actual design and implementation of the AO and $\mathrm{OO}$ versions have a significant influence on the resulting memory and performance numbers. Both versions have achieved the granularity, pluggability, and extensibility goals. One might, however, question if especially the design of the $\mathrm{OO}$ version is optimal with respect to the economy goal as well. Theoretically, it might be possible to find a better OO implementation that leads to lower memory demands.

As a matter of fact, it is impossible to proof that the design and implementation of some software is optimal with respect to runtime resource requirements. The authors have, however, profound experience in developing resource-minimal object-oriented product lines[11, 10,34]. There is, furthermore, quite some evidence that any OO version would lead to higher memory requirements than the AO version: As pointed out in the previous sections, runtime mechanisms have to be used with $\mathrm{OO}$ to reach the required flexibility which, as described in sections 3.5 and 4, induce overhead. Given that the OO version takes up to $138 \%$ more RAM and up to $91 \%$ more flash memory than the $\mathrm{C}$ and $\mathrm{AO}$ versions, it is hard to imagine that these differences could be levelled by a better $\mathrm{OO}$ implementation.

For the $\mathrm{AO}$ version the resource requirements are only minimal higher than for the $\mathrm{C}$ version. Hence, it can already be considered as "nearly optimal". In a recent study on the eCos operating system product line[1], we could show that the C-mechanism to reach configurability (conditional compilation), can be replaced by AOP concepts without any 
extra runtime and memory costs. In this study, we refactored several hundred \#ifdefblocks caused by configurable features into aspects[33].

\section{Related Work}

Several papers have been published that analyze and compare different implementation techniques of product line variabilities[23,36]. All authors come to the conclusion that AOP is a very promising approach, although still not widely used in the industrial practice[38]. In the middleware area several case studies showed that AOP allows software developers to scale features and footprint by supporting the static configuration of fine-grained configurable features[50,13,51]. All of these studies were conducted with AspectJ. In one case the authors clearly expressed that AspectC++ would be more appropriate for the embedded systems domain[28].

Other proposed implementation techniques were Generative Programming[16], the application of frame processors such as XVCL[29] or Angie[19], and Feature-Oriented Programming (FOP)[6]. All three techniques have in common with AOP that in a layered system design the higher-level layers can use special means to refine the behavior and/or structure of the lower-level layers. This dependency alignment [14] is a key to successful product line design. In comparison to frame processing and $\mathrm{C}++$ templatebased generative programming[17], the main advantage of the AOP approach is its obliviousness, i. e. refined layers don't have to be prepared for intervention by aspects. In comparison with refinements in FOP, the advantage of AOP is quantification, i. e. a single aspect can affect various different join points. Of course, FOP is much more than just the refinement mechanism. Therefore, the novel FeatureC++ language[5] is very promising, as it combines AOP and FOP.

When using OOP, the discussed refinement can only be achieved with virtual functions at the cost of dynamic binding. However, in many cases dynamic binding offers more flexibility than it is actually needed. A lot of work has been conducted to reduce the costs of virtual functions[3,20,8], but these optimizations are still not state of the art in current $\mathrm{C}++$ compilers.

Another alternative approach is to combine OOP with partial program evaluation tools like Tempo [15] and C-Mix [4], especially as partial evaluation has succesfully been applied in the context of system software [37]. However, the authors are not aware of any tools or concept papers that cover the combination of these techniques with OOP. Furthermore, the case studies on partial evaluation of system software focus on performance improvements. Some of these techniques increase the code size by keeping specialized and original code in memory at the same time or by applying dynamic compilation [42].

In the systems software area configurable software is often component-based. Popular examples are the OSKit[22,39] and TinyOS[7]. In both cases the requirements on performance and footprint can only be achieved with additional tool support. For example, Knit can be regarded as a weaver for OSKit components[43]. TinyOS is written in the NesC language[25]. Here the compiler performs a whole program transformation in order to optimize the system. In both cases the overhead, which is the result of the 
component model, is later removed by an additional tool. With the AOP approach an overhead is avoided from the beginning.

An interesting alternative to layered designs is Subject-Oriented Programming (SOP)[26, 40]. This technique is one of the roots of AOP. It supports system composition from slices instead of layers. In our embedded weather station case study, the sensors are in fact slices. By using the introduction mechanism of AspectC++, SOP was simulated.

\section{Summary And Conclusions}

The development of fine-grained and resource-efficient system software product lines requires means for separation of concerns that do not lead to an extra overhead in terms of memory and performance. Especially in the important domain of small embedded systems, where even a few additional bytes of code or data may lead to higher hardware costs, the inherent overhead of OOP is not acceptable for most developers. For this domain we propose AOP as an alternative.

In this paper, we compared an object-oriented with an aspect-oriented implementation of an configurable embedded software product line. We could show that the aspectoriented implementation induces significantly lower memory requirements than the object-oriented implementation, while providing similar or even better qualities with respect to separation of concerns. Although our results were produced with $\mathrm{C}++$ and AspectC++, similar effects should be achievable with any AOP approach that does not require additional runtime support and performs inlining of advice code. Advice code inlining also leads to an excellent performance. Our measurements have shown that the performance of the $\mathrm{AO}$ version was identical with the performance of our C-based reference implementation.

Overall, our results show that aspects provide very good separation of concerns in conjuction with high resource efficiency. This makes AOP well suited for the development of embedded system software product lines - where aspects beat objects.

Regarding future work, we will continue to evaluate costs and benefits of applying AOP to the operating systems domain. We are furthermore working on a benchmark suite that provides detailed results regarding the runtime and memory costs of AspectC++ features.

\section{Acknowledgments}

The authors would like to thank the anonymous reviewers for their helpful comments. We furthermore thank Danilo Beuche from pure::systems $\mathrm{GmbH}$ for leaving us the weather station hardware. Finally, a big thankyou goes to the editors, especially HansArno Jacobsen, whose suggestions helped us a lot to improve the quality and comprehensibility of this paper.

This work was partly supported by the German Research Council (DFG) under grant no. SCHR 603/4 and SP 968/2-1. 


\section{References}

1. eCos homepage. http://ecos.sourceware.org/.

2. OSEK/VDX standard. http://www.osek-vdx.org/.

3. Gerald Aigner and Urs Hölzle. Eliminating virtual function calls in $\mathrm{C}++$ programs. Technical Report TRCS95-22, Computer Science Department, University of California, Santa Barbara, December 1995.

4. Lars Ole Andersen. Program Analysis and Specialization for the C Programming Language. $\mathrm{PhD}$ thesis, DIKU, University of Copenhagen, May 1994. (DIKU report 94/19).

5. Sven Apel, Thomas Leich, Marko Rosenmller, and Gunter Saake. FeatureC++: On the symbiosis of feature-oriented and aspect-oriented programming. In Proceedings of the 4th International Conference on Generative Programming and Component Engineering (GPCE '05), Tallinn, Estonia, September 2005.

6. Don Batory. Feature-oriented programming and the AHEAD tool suite. In Proceedings of the 26th International Conference on Software Engineering (ICSE '04), pages 702-703. IEEE Computer Society, 2004.

7. UC Berkeley. TinyOS homepage. http://www.tinyos.net/.

8. David Bernstein, Yaroslav Fedorov, Sara Porat, Joseph Rodrigue, and Eran Yahav. Compiler optimization of $\mathrm{C}++$ virtual function calls. In Proceedings of the 2nd USENIX Conference on Object-Oriented Technologies and Systems (COOTS '96), Toronto, Canada, June 1996.

9. Danilo Beuche. Variant management with pure::variants. Technical report, pure-systems GmbH, 2003. http://www.pure-systems.com/.

10. Danilo Beuche, Abdelaziz Guerrouat, Holger Papajewski, Wolfgang Schröder-Preikschat, Olaf Spinczyk, and Ute Spinczyk. The PURE family of object-oriented operating systems for deeply embedded systems. In Proceedings of the 2nd IEEE International Symposium on Object-Oriented Real-Time Distributed Computing (ISORC '99), pages 45-53, St Malo, France, May 1999.

11. Danilo Beuche, Wolfgang Schröder-Preikschat, Olaf Spinczyk, and Ute Spinczyk. Streamlining object-oriented software for deeply embedded applications. In Proceedings of the 33rd International Conference on Technology of Object-Oriented Languages and Systems (TOOLS '00), pages 33-44, Mont Saint-Michel, France, June 2000.

12. Yvonne Coady, Gregor Kiczales, Michael Feeley, and Greg Smolyn. Using AspectC to improve the modularity of path-specific customization in operating system code. In Proceedings of the 3rd Joint European Software Engineering Conference and ACM Symposium on the Foundations of Software Engineering (ESEC/FSE '01), 2001.

13. Adrian Colyer, Andy Clement, Ron Bodkin, and Jim Hugunin. Using AspectJ for component integration in middleware. In Proceedings of the 18th ACM Conference on Object-Oriented Programming, Systems, Languages, and Applications (OOPSLA '03), pages 339-344, New York, NY, USA, 2003. ACM Press.

14. Adrian Colyer, Awais Rashid, and Gordon Blair. On the separation of concerns in program families. Technical Report COMP-001-2004, Lancaster University, 2004.

15. C. Consel, L. Hornof, R. Marlet, G. Muller, S. Thibault, and E.-N. Volanschi. Tempo: Specializing systems applications and beyond. ACM Computing Surveys, 30(3es), 1998.

16. Krysztof Czarnecki and Ulrich W. Eisenecker. Generative Programming. Methods, Tools and Applications. Addison-Wesley, May 2000.

17. Krzysztof Czarnecki and Ulrich W. Eisenecker. Synthesizing Objects. In R. Guerraoui, editor, Proceedings of the 13th European Conference on Object-Oriented Programming (ECOOP '99), number 1628 in Lecture Notes in Computer Science, pages 18-42, Lisbon, Portugal, 1999. Springer-Verlag. 
18. Jeffrey Dean, Craig Chambers, and David Grove. Selective specialization for object-oriented languages. In Proceedings of PLDI '95, La Jolla, CA, June 1995.

19. Delta Software Technology GmbH. Angie - an introduction, June 2005.

20. Karel Driesen and Urs Hölzle. The direct cost of virtual function calls in C++. In Proceedings of the 11th ACM Conference on Object-Oriented Programming, Systems, Languages, and Applications (OOPSLA '96), October 1996.

21. Bruno Dufour, Christopher Goard, Laurie Hendren, Clark Verbrugge, Oege de Moor, and Ganesh Sittampalam. Measuring the dynamic behaviour of AspectJ programs. In Proceedings of the 19th ACM Conference on Object-Oriented Programming, Systems, Languages, and Applications (OOPSLA '04), pages 150-169, New York, NY, USA, 2004. ACM Press.

22. Bryan Ford, Godmar Back, Greg Benson, Jay Lepreau, Albert Lin, and Olin Shivers. The flux OSKit: A substrate for Kernel and language research. In Proceedings of the 17th ACM Symposium on Operating Systems Principles (SOSP '97), ACM Operating Systems Review, pages 38-51. ACM Press, October 1997.

23. Cristina Gacek and Michalis Anastasopoules. Implementing product line variabilities. In Proceedings of 2001 Symposium on Software Reusability: Putting Software Reuse in Context, pages 109-117. ACM Press, 2001.

24. Erich Gamma, Richard Helm, Ralph Johnson, and John Vlissides. Design Patterns: Elements of Reusable Object-Oriented Software. Addison-Wesley, 1995.

25. David Gay, Philip Levis, Robert von Behren, Matt Welsh, Eric Brewer, and David Culler. The nesC language: A holistic approach to networked embedded systems. In Proceedings of the ACM SIGPLAN Conference on Programming Language Design and Implementation (PLDI '03), pages 1-11, San Diego, CA, USA, 2003. ACM Press.

26. William Harrison and Harold Ossher. Subject-oriented programming - a critique of pure objects. In Proceedings of the 8th ACM Conference on Object-Oriented Programming, Systems, Languages, and Applications (OOPSLA '93), pages 411-428, September 1993.

27. Michael Haupt and Mira Mezini. Micro-measurements for dynamic aspect-oriented systems. In NetObjectDays (NODe '04), volume 3263 of Lecture Notes in Computer Science, pages 81-96, Erfurt, Germany, September 2004. Springer-Verlag.

28. Frank Hunleth and Ron Cytron. Footprint and feature management using aspect-oriented programming techniques. In Proceedings of the 2002 Joint Conference on Languages, Compilers and Tools for Embedded Systems \& Soft. and Compilers for Embedded Systems (LCTES/SCOPES '02), pages 38-45, Berlin, Germany, June 2002. ACM Press.

29. Stan Jarzabek and Hongyu Zhang. XML-based method and tool for handling variant requirements in domain model. In Proceedings of the 5th IEEE International Symposium on Requirements Engineering (RE '01), pages 116-123, Toronto, Canada, August 2001. IEEE Computer Society Press.

30. J. M. Kahn, R. H. Katz, and K. S. J. Pister. Next century challenges: Mobile networking for "smart dust". In International Conference on Mobile Computing and Networking (MOBICOM '99), pages 271-278, 1999.

31. Gregor Kiczales, Erik Hilsdale, Jim Hugunin, Mik Kersten, Jeffrey Palm, and William G. Griswold. An overview of AspectJ. In J. Lindskov Knudsen, editor, Proceedings of the 15th European Conference on Object-Oriented Programming (ECOOP '01), volume 2072 of Lecture Notes in Computer Science, pages 327-353. Springer-Verlag, June 2001.

32. Daniel Lohmann, Georg Blaschke, and Olaf Spinczyk. Generic advice: On the combination of AOP with generative programming in AspectC++. In G. Karsai and E. Visser, editors, Proceedings of the 3rd International Conference on Generative Programming and Component Engineering (GPCE '04), volume 3286 of Lecture Notes in Computer Science, pages 55-74. Springer-Verlag, October 2004. 
33. Daniel Lohmann, Fabian Scheler, Reinhard Tartler, Olaf Spinczyk, and Wolfgang SchröderPreikschat. A quantitative analysis of aspects in the eCos kernel. In Proceedings of the EuroSys 2006 Conference (EuroSys '06), pages 191-204. ACM Press, April 2006.

34. Daniel Lohmann, Wolfgang Schröder-Preikschat, and Olaf Spinczyk. On the design and development of a customizable embedded operating system. In Proceedings of the SRDS Workshop on Dependable Embedded Systems (SRDS-DES '04), pages 1-6. IEEE Computer Society, October 2004.

35. Daniel Lohmann and Olaf Spinczyk. On typesafe aspect implementations in C++. In F. Geschwind, U. Assmann, and O. Nierstrasz, editors, Proceedings of Software Composition 2005 (SC '05), volume 3628 of Lecture Notes in Computer Science, pages 135-149, Edinburgh, UK, April 2005. Springer-Verlag.

36. Mira Mezini and Klaus Ostermann. Variability management with feature-oriented programming and aspects. In Proceedings of ACM SIGSOFT '04 / FSE-12, November 2004.

37. G. Muller, E.N. Volanschi, and R. Marlet. Scaling up partial evaluation for optimizing the sun commercial rpc protocol. In ACM SIGPLAN Symposium on Partial Evaluation and Semantics-Based Program Manipulation, pages 116-125, Amsterdam, The Netherlands, June 1997. ACM Press.

38. Dirk Muthig and Thomas Patzke. Generic implementation of product line components. In NetObjectDays (NODe '02), volume 2591 of Lecture Notes in Computer Science, pages 313-329, Erfurt, Germany, October 2003. Springer-Verlag.

39. University of Utah. OSKit homepage. http://www.cs.utah.edu/flux/oskit.

40. Harold Ossher and Peri Tarr. Using multidimensional separation of concerns to (re)shape evolving software. Communications of the ACM, pages 43-50, October 2001.

41. D. L. Parnas. Some hypothesis about the uses hierarchy for operating systems. Technical report, TH Darmstadt, Fachbereich Informatik, 1976.

42. Calton Pu, Henry Massalin, and John Ioannidis. The Synthesis kernel. Computing Systems, 1(1):11-32, Winter 1988.

43. Alastair Reid, Matthew Flatt, Leigh Stoller, Jay Lepreau, and Eric Eide. Knit: Component composition for systems software. In Proceedings of the 4th Symposium on Operating Systems Design and Implementation (OSDI 'O0), pages 347-360, San Diego, CA, USA, October 2000. Usenix Association.

44. O. Rohlik, A. Pasetti, V. Cechticky, and I. Birrer. Implementing adaptability in embedded software through aspect oriented programming. In Proceedings of Mechatronics \& Robotics (MechRob '04), Aachen, Germany, September 2004. IEEE Computer Society Press.

45. Wolfgang Schröder-Preikschat, Daniel Lohmann, Wasif Gilani, Fabian Scheler, and Olaf Spinczyk. Static and dynamic weaving in system software with AspectC++. In Yvonne Coady, Jeff Gray, and Raymond Klefstad, editors, Proceedings of the 39th Hawaii International Conference on System Sciences (HICSS '06) - Mini-Track on Adaptive and Evolvable Software Systems. IEEE Computer Society Press, 2006.

46. Olaf Spinczyk, Daniel Lohmann, and Matthias Urban. Advances in AOP with AspectC++. In Hamido Fujita and Mohamed Mejri, editors, New Trends in Software Methodologies, Tools and Techniques (SoMeT '05), number 129 in Frontiers in Artificial Intelligence and Applications, pages 33-53, Tokyo, Japan, September 2005. IOS Press.

47. David Tennenhouse. Proactive computing. Communications of the ACM, pages 43-45, May 2000.

48. Mark Weiser. The computer for the 21st centrury. Scientific American, 265(3):94-104, 1991.

49. Trevor Young and Gail Murphy. Using AspectJ to build a product line for mobile devices. Chicago, Illinois, March 2005. AOSD '05 Demo.

50. Charles Zhang, Depend Gao, and Hans-Arno Jacobsen. Generic middleware substrate through modelware. In Proceedings of the ACM/IFIP/USENIX 6th International Middleware Conference (Middleware '05), Grenoble, France, 2005. ACM Press. 
51. Charles Zhang and Hans-Arno Jacobsen. Refactoring middleware with aspects. IEEE Transactions on Parallel and Distributed Systems, 14(11), November 2003. 